\title{
Successful Laparoscopic Resection of Gastric Lymphangioma Under the Intraoperative Guidance of Indocyanine Green Fluorescence Imaging: A Case Report
}

Ryosuke Umino ( $\nabla$ ryosukeumino@gmail.com )

Toranomon Hospital: Toranomon Byoin https://orcid.org/0000-0001-6659-2027

Masayuki Urabe

Toranomon Hospital: Toranomon Byoin

Yu Ohkura

Toranomon Hospital: Toranomon Byoin

Akikazu Yago

Toranomon Hospital: Toranomon Byoin

Shusuke Haruta

Toranomon Hospital: Toranomon Byoin

Masaki Ueno

Toranomon Hospital: Toranomon Byoin

Harushi Udagawa

Toranomon Hospital: Toranomon Byoin

\section{Case report}

Keywords: Gastric lymphangioma, gastrectomy, indocyanine green

Posted Date: December 2nd, 2020

DOl: https://doi.org/10.21203/rs.3.rs-117730/v1

License: (c) (1) This work is licensed under a Creative Commons Attribution 4.0 International License.

Read Full License

Version of Record: A version of this preprint was published at Asian Journal of Endoscopic Surgery on April 27th, 2021. See the published version at https://doi.org/10.1111/ases.12946. 


\section{Abstract}

Background: Gastric lymphangioma (GLA) is an extremely rare tumor without an established therapeutic strategy. Surgical resection is considered the mainstay of treatment, although there is a high risk of local recurrence if negative margins are not achieved. Thus, it would be useful to develop tools and strategies to achieve safe and complete resection. We describe our experience with the first case involving GLA resection under the intraoperative guidance of indocyanine green (ICG) fluorescence imaging, which allowed us to achieve limited but complete resection with negative margins.

Case Presentation: A 51-year-old Japanese man with a history of alcoholic liver disease underwent routine abdominal ultrasonography, which incidentally detected a 20-mm tumor adjacent to the lesser curvature of the stomach. The mass was suspected to be GLA based on its polycystic appearance. After a 16-month monitoring period, the patient was referred to our hospital for further assessment because of tumor growth and involvement of the left gastric artery. We selected surgical resection to facilitate a pathological diagnosis and treatment of potential invasion to the surrounding organs. Intraoperative use of a ICG navigation system revealed lymphatic drainage from the tumor, which we used to help determine the optimal excision line and minimize the loss of gastric volume. Pathological examination confirmed complete resection with negative margins and supported a diagnosis of lymphangioma.

Conclusions: We performed laparoscopic radical resection of GLA under guidance from intraoperative ICG fluorescence imaging, which allowed us to maximize residual gastric volume. Although further cases are needed to validate this strategy, it may be useful for guiding the resection of GLA.

\section{Background}

Gastric lymphangioma (GLA) is a very rare tumor and we are only aware of approximately 100 reported cases of GLA [1, 2]. Small GLAs can be followed without direct treatment, although careful monitoring is needed because of its oncological potential. However, surgical resection should be considered when GLArelated problems are considered probable, such as involvement of adjacent organs, gastrointestinal obstruction, tumor rupture, and/or bleeding. Although surgical resection is the mainstay of radical treatment for GLA [3], previous authors have suggested that margin-free resection is difficult because the tumor tends to invade the surrounding tissues with ambiguous boundaries, which produces a high rate of local recurrence $[4,5]$. Complete resection should be targeted to prevent GLA recurrence, although it is also important to maintain gastric functionality, as GLA is potentially benign as long as it is completely removed [4]. Thus, it would be useful to develop tools and strategies to achieve safe but oncologically radical resection.

Indocyanine green (ICG) fluorescent imaging has recently been used during gastrointestinal surgery to evaluate lymphovascular flow and to help clarify the tumor site [6-8]. Information from intraoperative ICG-assisted angiography or lymphography can also help visualize the tumor and guide the excision range without damaging uninvolved vessels. We report our experience with a case of successfully 
resected GLA that was guided by intraoperative ICG fluorescence imaging, which resulted in a complete radical resection and preserved residual gastric volume.

\section{Case Presentation}

A 51-year-old Japanese man was undergoing medical treatment for alcoholic liver injury. Routine abdominal ultrasonography incidentally identified a $20-\mathrm{mm}$ mass located between the lateral segment of the liver and the stomach. Magnetic resonance imaging (MRI) revealed a multi-cystic lesion with hyperdensity on T2-weighted imaging, which seemed to contact the lesser curvature of the stomach (Fig. 1a). The mass was suspected to be GLA based on it apparently originating from the gastric wall and its polycystic appearance. After a 16-month monitoring period, the tumor had grown and MRI revealed intraluminal infiltration (Fig. 1b). Contrast-enhanced computed tomography also revealed that the tumor extended to the lymph nodes around the left gastric artery (LGA), although the LGA itself was intact (Fig. 2). Positron emission tomography revealed no abnormal uptake of $\mathrm{F}^{18}$-fluorodeoxyglucose within the tumor. An upper gastrointestinal fiberscope examination revealed extrinsic compression to the lesser anterior wall of the stomach (Fig. 3a) and endoscopic ultrasonography revealed a cystic lesion that had partially infiltrated the gastric wall (Fig. 3b). The results of a physical examination were unremarkable, and laboratory tests revealed no abnormal findings aside from elevated serum transaminase concentrations (AST: $145 \mathrm{U} / \mathrm{mL}, A L T: 77 \mathrm{U} / \mathrm{mL}$ ), which were consistent with alcoholic liver injury.

We selected surgical resection based on the expansive and infiltrative tumor, which would also facilitate a pathological diagnosis and treat potential invasion to the surrounding organs. Before the surgery, we prepared an ICG fluorescence navigation system to assess the border between the tumor and normal tissues. Off-label use of ICG was approved by our institutional review board and the patient provided informed consent. The intraoperative findings revealed that the mass at the lesser curvature of the stomach was soft and elastic (Fig. 4a). A subserosal injection of ICG solution (Diagnogreen ${ }^{\circledR}$, DaiichiSankyo Pharma, Tokyo, Japan) was initially performed at the anterior wall of the stomach, although this failed to confirm staining of the tumor or the lymphatic vessels. An additional injection near the tumor revealed lymphatic flow (Fig. 4b] and the first incision of the lesser omentum was initiated from the angular incisure to the cardia. However, the approximately 5 -cm tumor was tightly adhered to the stomach and ICG lymphography using an intraoperative imaging system (1588 AIM Stryker, Tokyo, Japan) revealed lymphatic vessel drainage from the tumor toward the LGA (Fig. 4c]. Thus, the lymphatic vessels surrounding the LGA were ligated to prevent lymphatic drainage into the para-aortic nodes (Fig. 4d]. The stomach was then stapled at the superior and inferior edges of the tumor, and segmental gastrectomy with a delta-shaped anastomosis was performed [9]. The ICG lymphography subsequently revealed no fluorescence around the stomach (Fig. 4e,f).

Histopathological examination of the resected specimen revealed that the tumor $(95 \mathrm{~mm} \times 60 \mathrm{~mm} \times$ $25 \mathrm{~mm}$ ) arose mainly from the subserous tissues (Fig. 5a), and infiltration of dilated lymphatic vessels was observed in the subserous layer (Fig. 5b). The surgical margins were negative for GLA. The patient 
was discharged 10 days after surgery and his postoperative course has been uneventful, with no evidence of recurrence at 24 months after surgery.

\section{Discussion}

We encountered a case involving a GLA that arose from the lesser curvature of the stomach and performed laparoscopic radical resection under the intraoperative guidance of ICG fluorescence imaging, which maximized the residual gastric volume. Lymphangioma is a benign tumor that is typically observed in lymphatic rich areas, such as the head, neck, axilla, and mediastinum, and is most common in children [10], with $<1 \%$ of lymphangioma cases involving the gastrointestinal tract and/or the abdominal cavity. Most reports have described GLA as asymptomatic [11] and it is typically identified incidentally during check-ups or other medical treatment. Aggressive treatment is generally not needed for GLA, as long as it remains small and does not cause problematic symptoms. However, surgical resection is an option if the tumor grows sufficiently large to invade or compress the adjacent organs. In our case, the tumor had rapidly grown during a 16-month monitoring period, although the patient did not experience any related symptoms. Thus, we decided to attempt resection before more extended surgery might be required.

Lymphangioma has a tendency to invade the surrounding tissues and frequently recurs at the surgical site [12], which highlights the importance of margin-free resection in minimizing the risk of recurrence. However, surgeons often struggle to balance oncologically radical resection and preservation of gastric function in cases involving stomach tumors. In this context, ICG fluorescence imaging has been widely employed to guide various types of gastroenterological, neural, and plastic surgeries [6-8]. A real-time intraoperative detection system can help the surgeon achieve complete resection by visualizing a clear boundary between the tumor and normal tissues, while minimizing gastric volume loss. Furthermore, lymphangioma has dilated lymphatic vessels and is not connected to the normal lymphatic system [13], which suggests that ICG fluorescence imaging may be useful during surgery for lymphangioma. To the best of our knowledge, only one reported case has involved intraoperative ICG lymphography during treatment for lymphangioma in the abdominal wall [13], and ours appears to be the first reported case involving ICG fluorescence imaging during surgery for GLA. We used ICG imaging to clarify that the GLA had extended to the LGA and that there was no residual fluorescence after the resection. However, additional information is needed regarding the optimal ICG dosage and injection site.

\section{Conclusions}

We encountered a lymphangioma that arose from the lesser curvature of the stomach, and successfully resected the lesion under guidance from ICG fluorescence imaging. This innovative technique helped us achieve complete resection of the tumor with limited loss of gastric volume. While this procedure may provide significant benefits to patients who are undergoing resection of GLA, additional cases should be accumulated to validate our findings. 


\section{Declarations}

Ethics approval and consent to participate: The off-label use of indocyanine green was approved by our institutional review board. The patient provided informed consent for this treatment strategy.

Consent for publication: Not applicable.

Availability of data and materials: All relevant data are included in the published report.

Competing interests: None.

Funding: None.

Authors' contributions: All authors contributed to this case report. The first draft of the manuscript was written by RU. MU revised the manuscript. SH supervised the writing of the manuscript. All authors commented on previous versions and approved the final manuscript.

Acknowledgements: None.

\section{References}

1. Palmer ED. Benign intramural tumors of the stomach: a review with special reference to gross pathology. Medicine. 1951;30:81-181.

2. Matsushita A, Yuasa N, Miyake H, Nagai H, Nagao T, Fujino M. Gastric lymphangioma coexisting with mucosal gastric cancer: a rare case report. Clin J Gastroenterol. 2020;13:46-9.

3. Losanoff JE, Richman BW, El-Sherif A, Rider KD, Jones JW. Mesenteric cystic lymphangioma. J Am Coll Surg. 2003;196:598-603.

4. Amodeo I, Cavallaro G, Raffaeli G, Colombo L, Fumagalli M, Cavalli R, et al. Abdominal cystic lymphangioma in a term newborn: A case report and update of new treatments. Medicine. 2017;96:e5984.

5. Hancock BJ, St-Vil D, Luks FI, Di Lorenzo M, Blanchard H. Complications of lymphangiomas in children. J Pediatr Surg. 1992;27:220-6.

6. Kobayashi Y, Kawaguchi Y, Kobayashi K, Mori K, Arita J, Sakamoto Y, et al. Portal vein territory identification using indocyanine green fluorescence imaging: Technical details and short-term outcomes. J Surg Oncol. 2017;116:921-31.

7. Zelken JA, Tufaro AP. Current trends and emerging future of indocyanine green usage in surgery and oncology: An update. Ann Surg Oncol. 2015;22(Suppl3):1271-83.

8. Zhang YM, Shi R, Hou JC, Liu ZR, Cui ZL, Li Y, et al. Liver tumor boundaries identified intraoperatively using real-time indocyanine green fluorescence imaging. J Cancer Res Clin Oncol. 2016;143:51-8.

9. .Kanaya S, Gomi T, Momoi H, Tamaki N, Isobe H, Katayama T, et al. Delta-Shaped Anastomosis in Totally Laparoscopic Billroth I Gastrectomy: New Technique of Intraabdominal 
Gastroduodenostomy. J Am Coll Surg. 2002;195:284-7.

10. Makni A, Chebbi F, Fetirich F, Ksantini R, Bedioui H, Jouini M, et al. Surgical management of intraabdominal cystic lymphangioma. Report of 20 cases. World J Surg. 2012;36:1037-43.

11. Kang BH, Hur H, Joung YS, Kim DK, Kim YB, Ahn CW, et al. Giant mesenteric cystic lymphangioma originating from the lesser omentum in the abdominal cavity. J Gastric Cancer. 2011;11:243-7.

12. Taibi A, Derbal S, Mathonnet M, Durand Fontanier S. Cystic mass appended to the greater curvature of the stomach? Cystic lymphangioma of the greater omentum. J Visc Surg. 2020;157:443-5.

13. Shirota C, Hinoki A, Takahashi M, Tanaka Y, Tainaka T, Sumida W, et al. New navigation surgery for resection of lymphatic malformations using indocyanine green fluorescence imaging. Am J Case Rep. 2017;18:529-31.

\section{Figures}
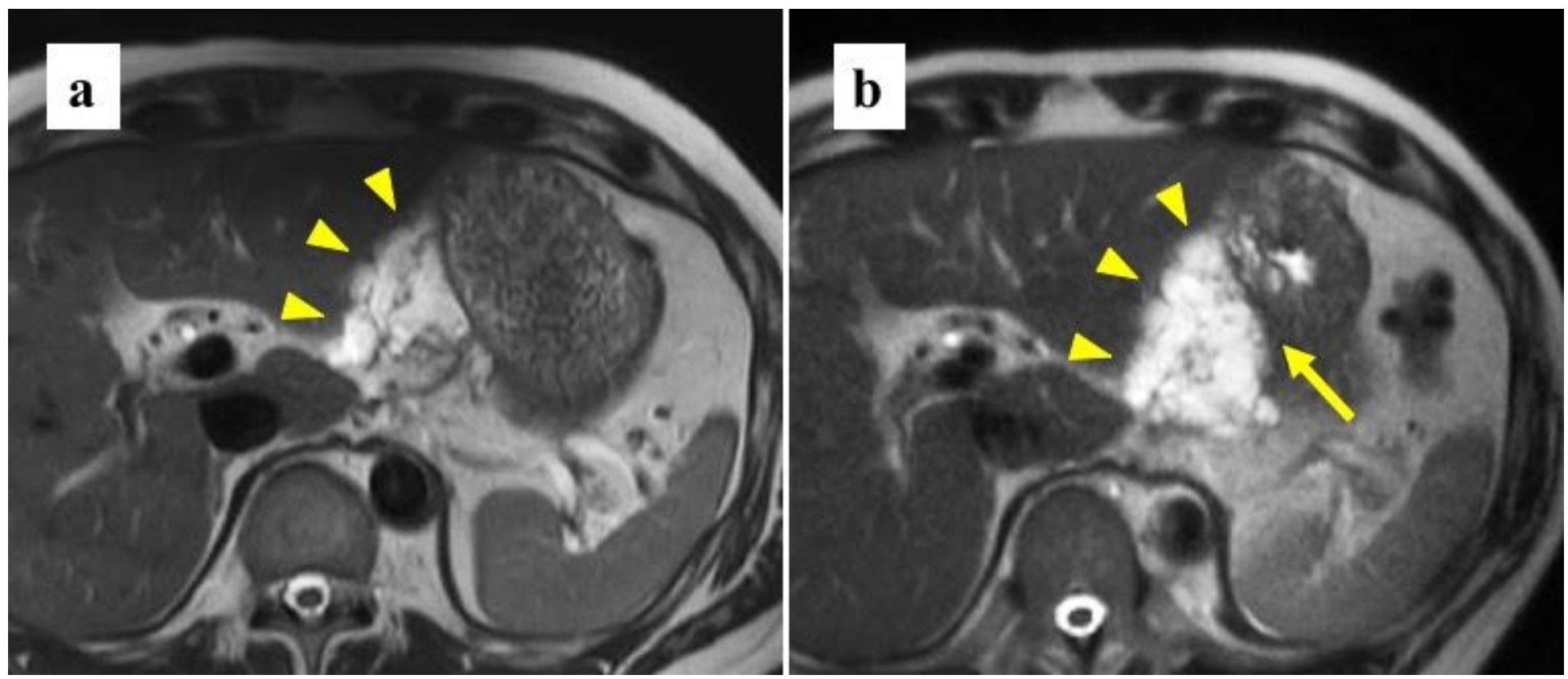

Figure 1

a: At the initial examination, magnetic resonance imaging revealed a multi-cystic lesion with hyperdensity on T2-weighted imaging at the lesser curvature of the stomach (yellow head). b: After a 16-month followup, the tumor had grown and infiltrated the stomach (yellow arrow). 

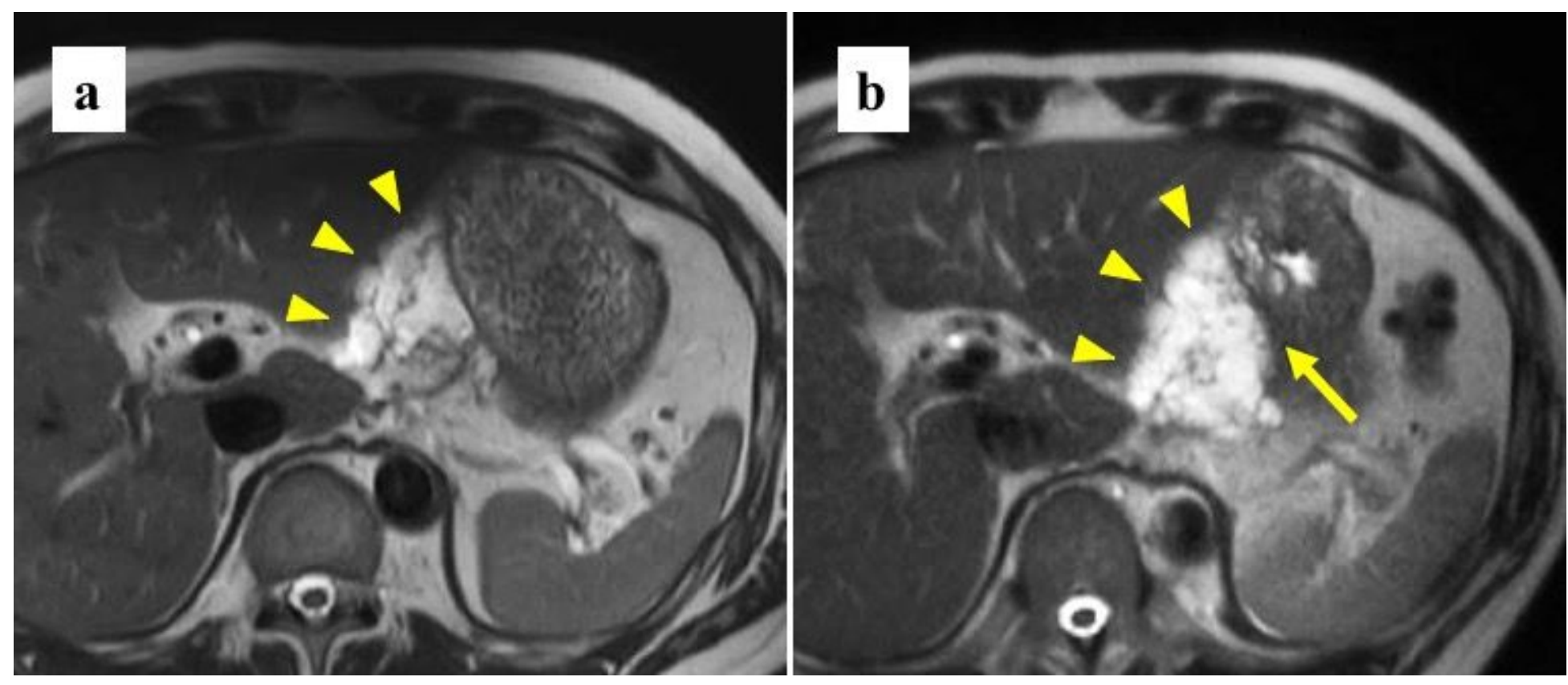

\section{Figure 1}

a: At the initial examination, magnetic resonance imaging revealed a multi-cystic lesion with hyperdensity on T2-weighted imaging at the lesser curvature of the stomach (yellow head). b: After a 16-month followup, the tumor had grown and infiltrated the stomach (yellow arrow).

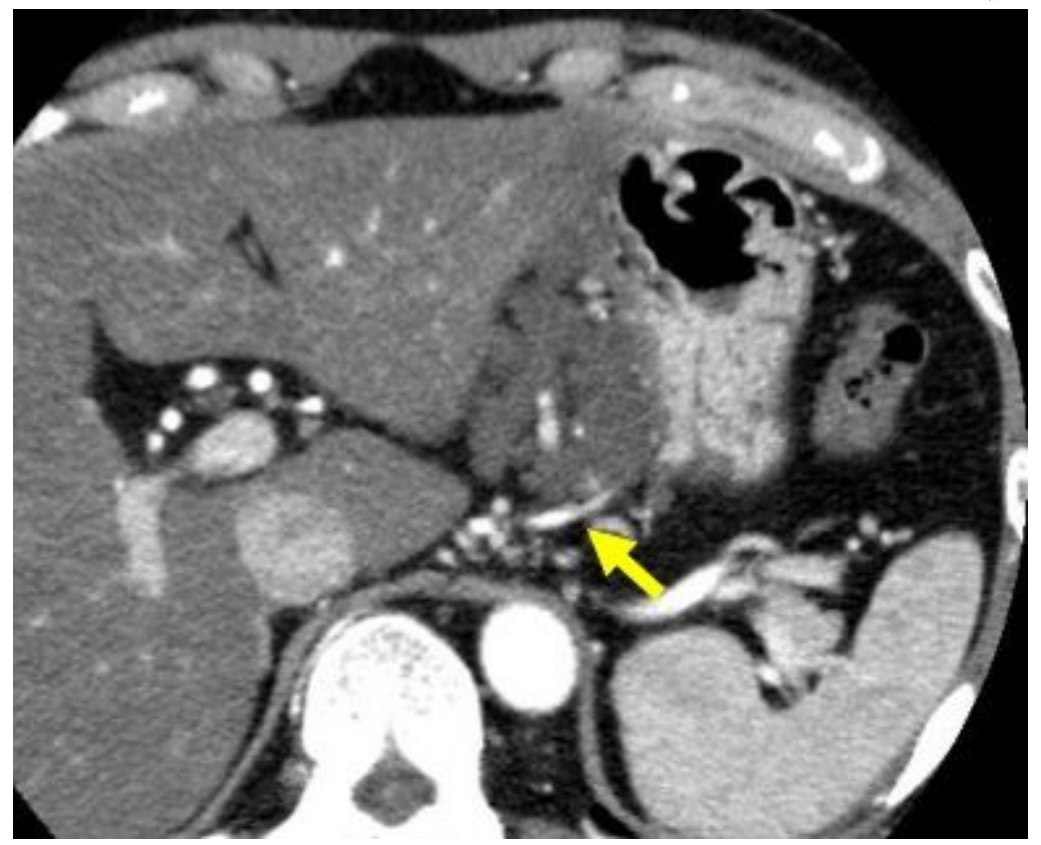

Figure 2

a: Computed tomography revealed the tumor extending to the left gastric artery lymph nodes (yellow arrow). b: Positron emission tomography-computed tomography revealed no fluorodeoxyglucose uptake. 


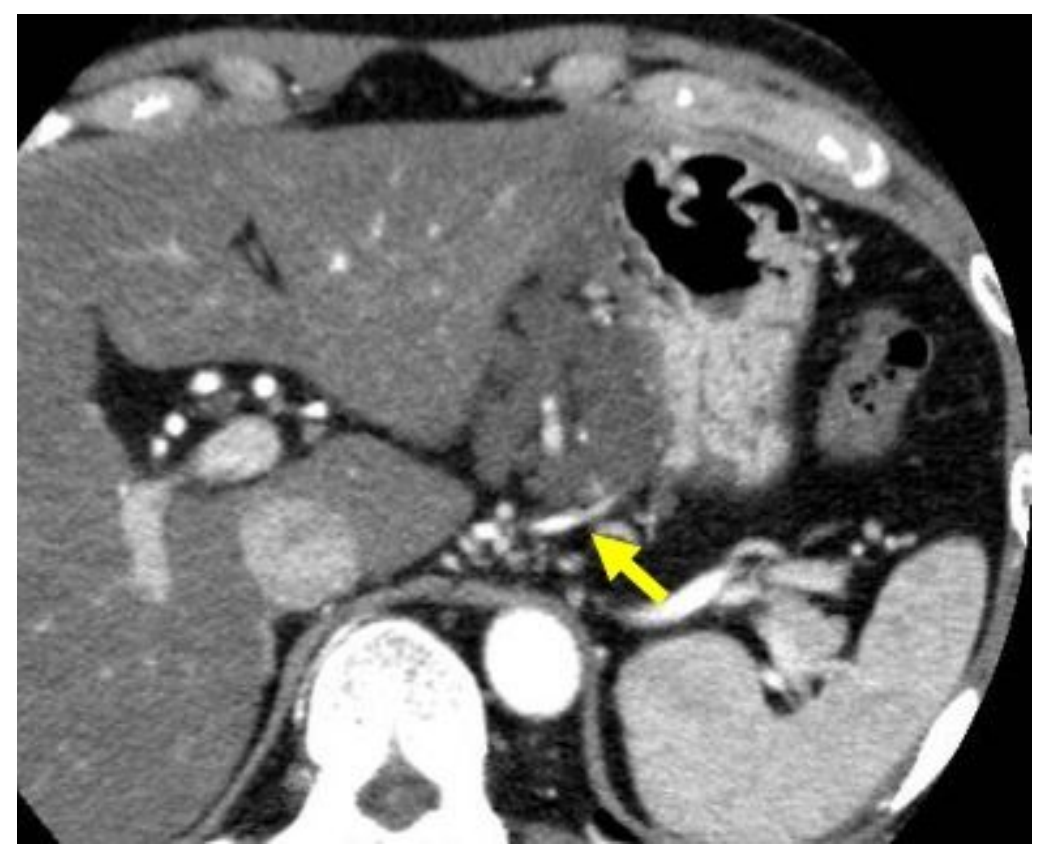

\section{Figure 2}

a: Computed tomography revealed the tumor extending to the left gastric artery lymph nodes (yellow arrow). b: Positron emission tomography-computed tomography revealed no fluorodeoxyglucose uptake.
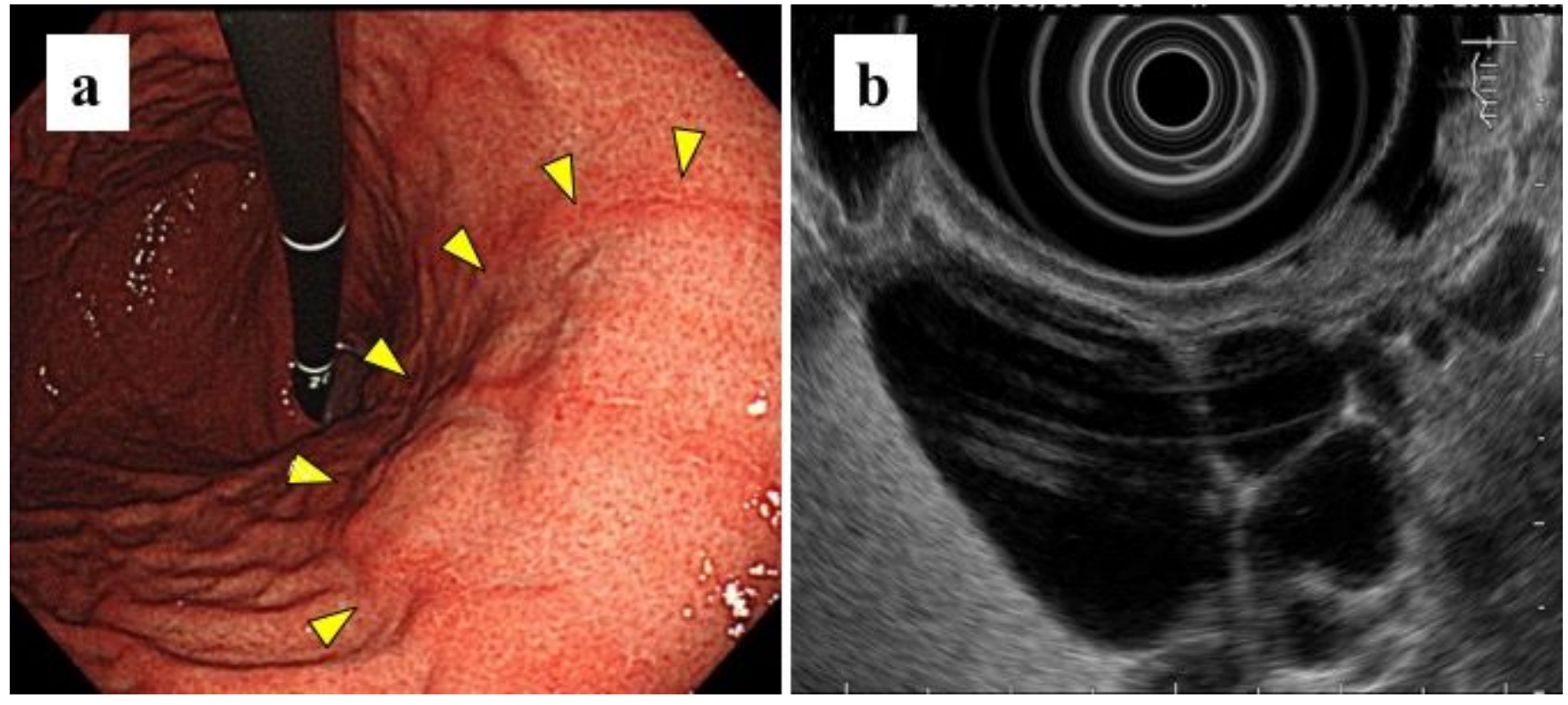

\section{Figure 3}

a: Examination using an upper gastrointestinal fiberscope revealed extrinsic compression of the lesser anterior wall of the stomach (yellow head). b: Endoscopic ultrasonography revealed a cystic lesion adjacent to the gastric wall. 

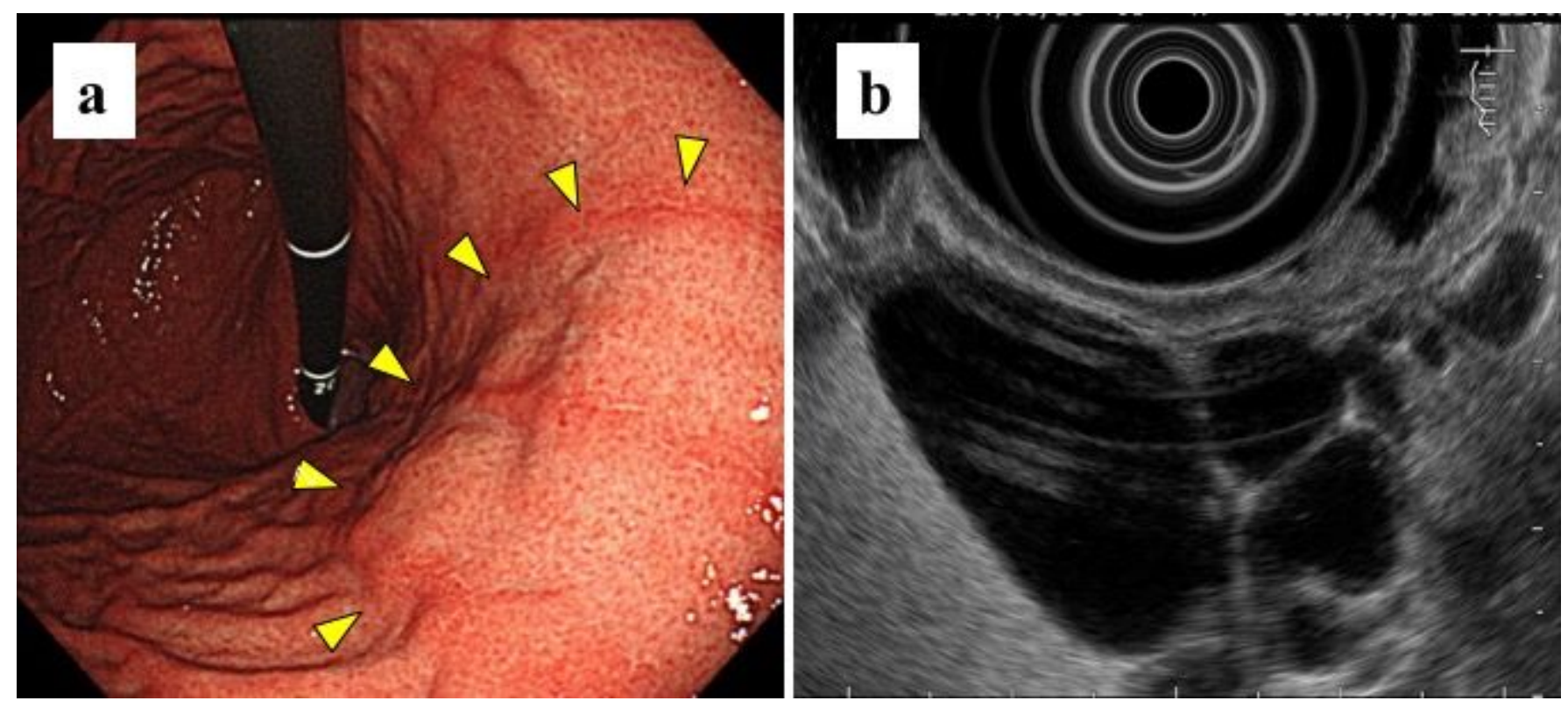

\section{Figure 3}

a: Examination using an upper gastrointestinal fiberscope revealed extrinsic compression of the lesser anterior wall of the stomach (yellow head). b: Endoscopic ultrasonography revealed a cystic lesion adjacent to the gastric wall.

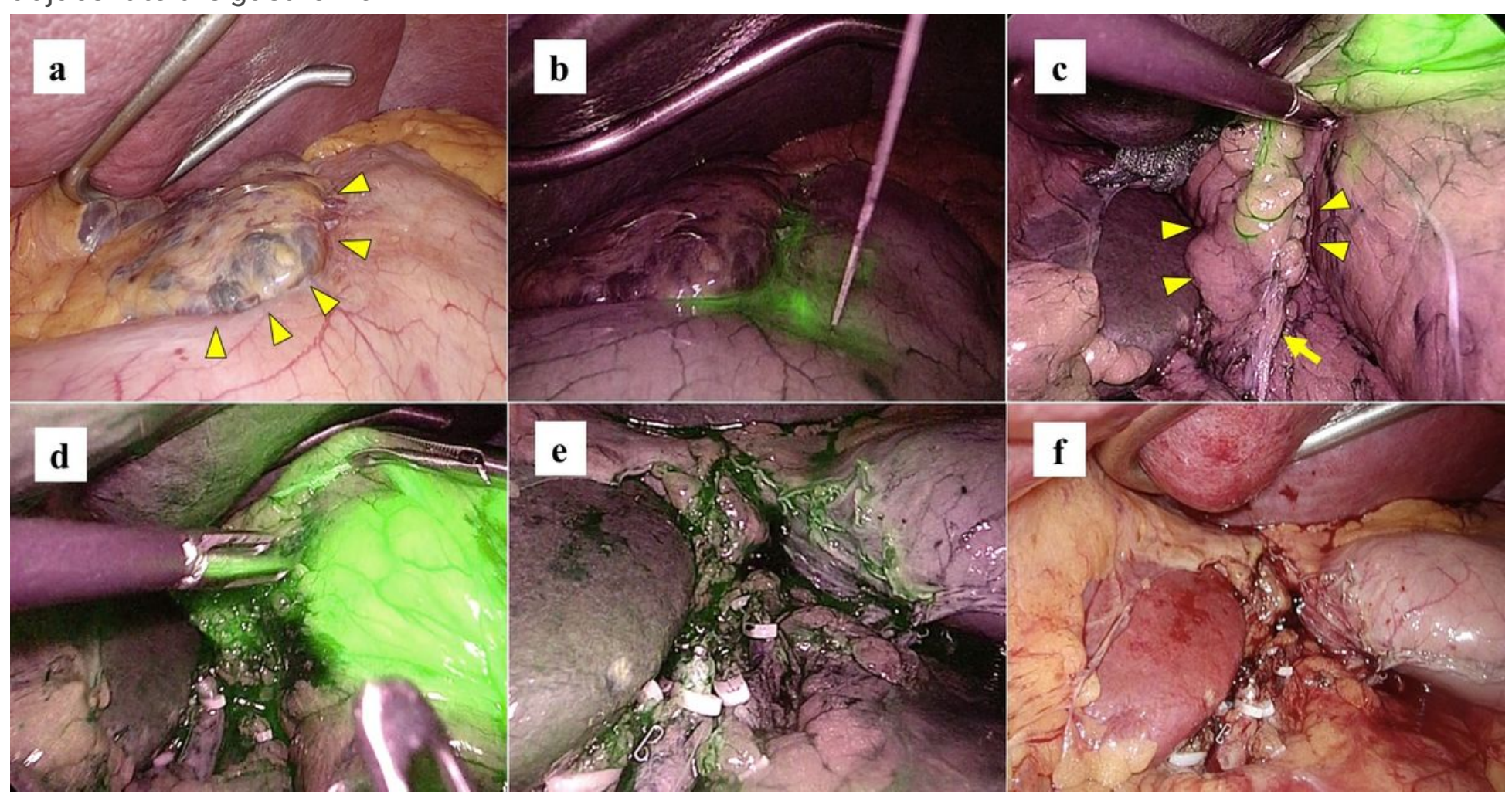

\section{Figure 4}

Intraabdominal findings. a: The tumor at the lesser curvature of the stomach (yellow head). b: The injection of indocyanine green. c: The indocyanine green fluorescence imaging revealed that the tumor (yellow head) extended to the confluence of the left gastric artery (yellow arrow). d: Lymphatic vessels 
surrounding the left gastric artery were ligated. e, f: No residual tumor was observed and the indocyanine green fluorescence imaging revealed no fluorescence.

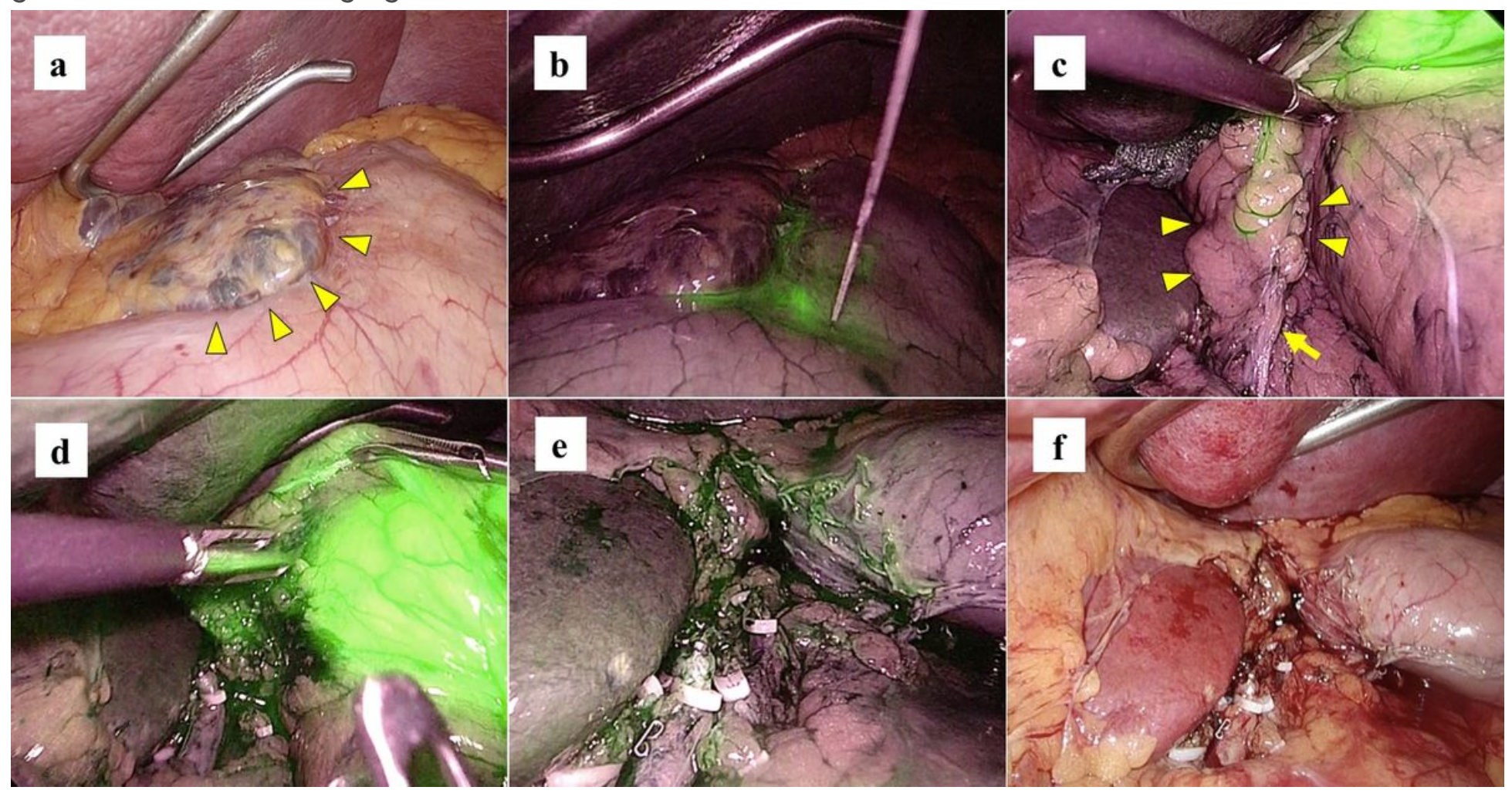

\section{Figure 4}

Intraabdominal findings. a: The tumor at the lesser curvature of the stomach (yellow head). b: The injection of indocyanine green. c: The indocyanine green fluorescence imaging revealed that the tumor (yellow head) extended to the confluence of the left gastric artery (yellow arrow). d: Lymphatic vessels surrounding the left gastric artery were ligated. e, f: No residual tumor was observed and the indocyanine green fluorescence imaging revealed no fluorescence.
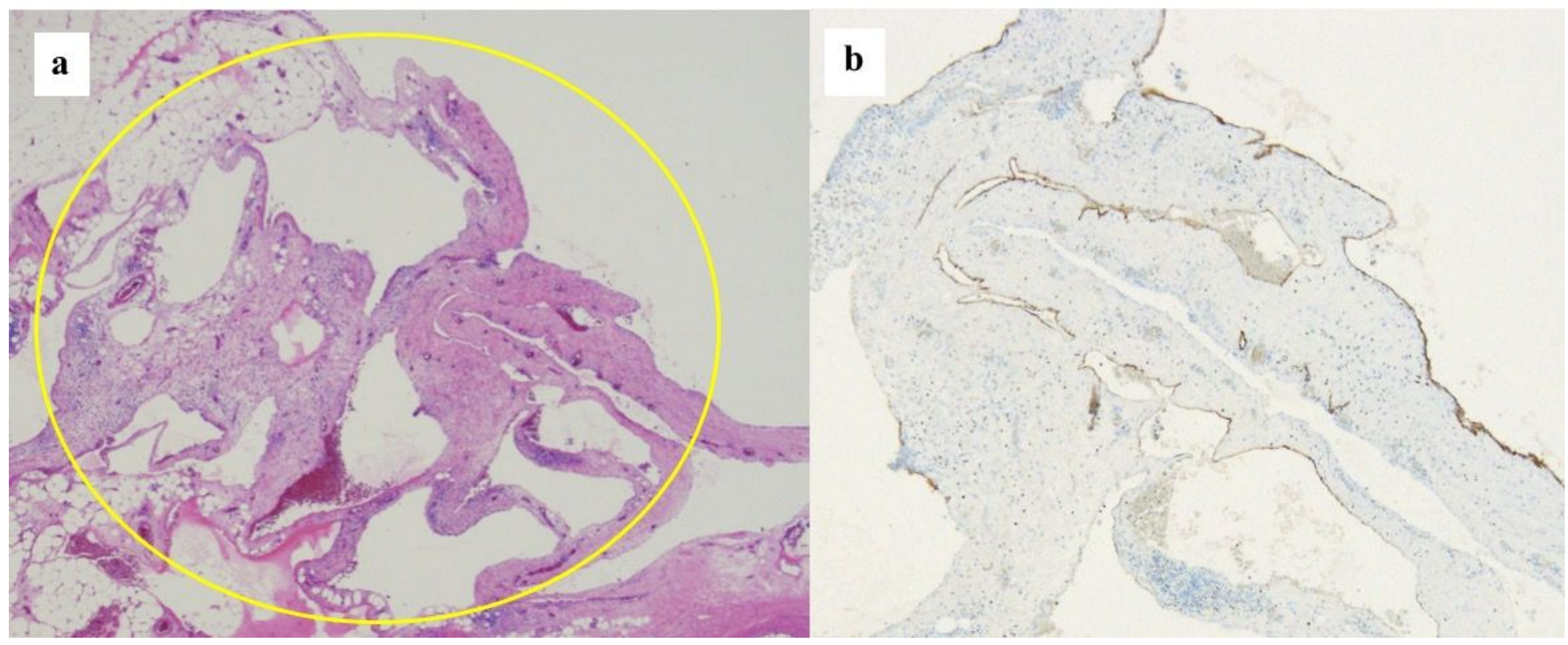


\section{Figure 5}

a: A histological examination revealed that the tumor arose from the subserosal layer (yellow circle). b: Infiltration of the dilated lymphatic vessels was observed in the subserosal layer (D2-40 positive).
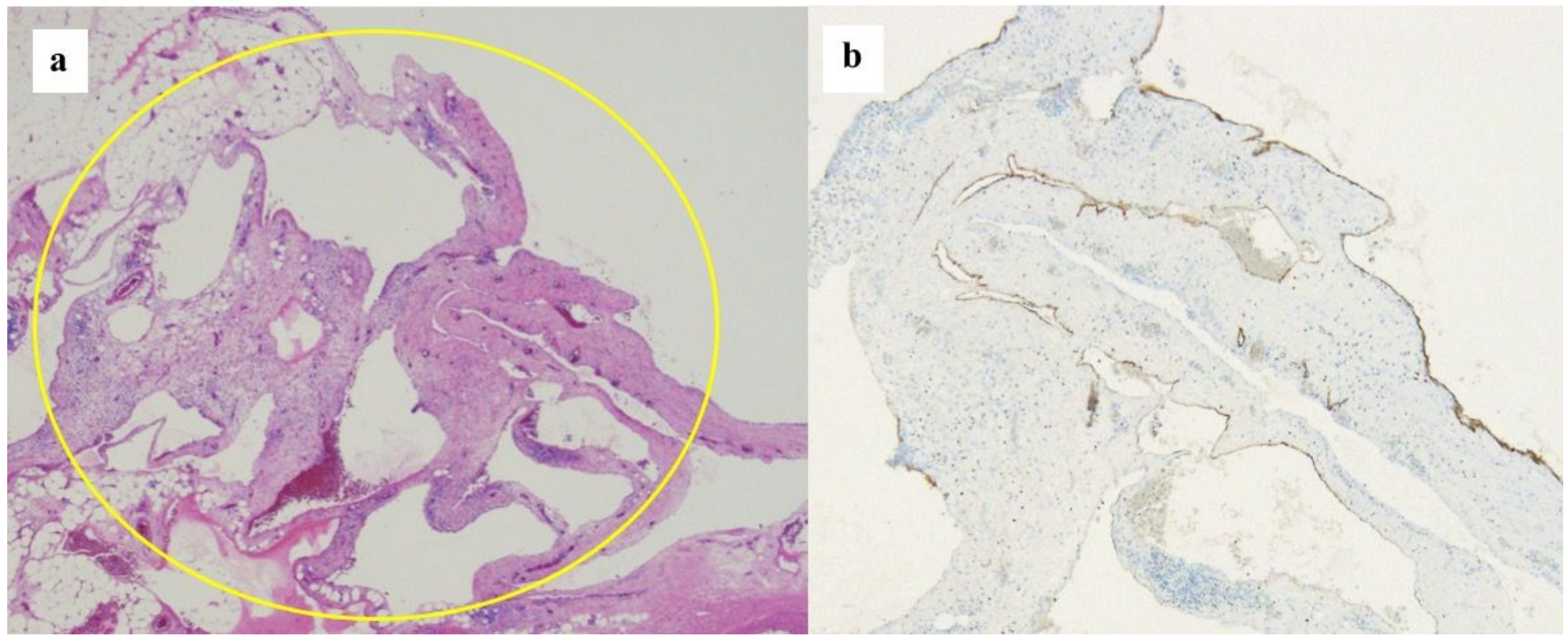

Figure 5

a: A histological examination revealed that the tumor arose from the subserosal layer (yellow circle). b: Infiltration of the dilated lymphatic vessels was observed in the subserosal layer (D2-40 positive). 